\title{
Development and test of distributed ledger technologies applications in a microgrid distributed control
}

\author{
D. Coll-Mayor ${ }^{1}$, A. Notholt ${ }^{1}$ \\ ${ }^{1}$ School of Engineering \\ Reutlingen University \\ Alteburgstr. 15072762 Reutlingen (Germany) \\ Phone/Fax number: +49 (7121) 271-7139, \\ e-mail: Debora.Coll-Mayor@Reutlingen-University.de, Antonio.Notholt@Reutlingen-University.de,
}

\begin{abstract}
This paper presents the preliminary results of a set of research projects being developed at the distributed resources laboratory at the University of Reutlingen. The main aim of these projects is to couple distributed ledger technologies (DLTs) with distributed control of microgrids. Firstly, a DLT based solution for a local market platform has been developed. This enables end customers to participate in new local micro-energy-markets by providing them with a distributed, decentralized, transparent and secure Peer to Peer (P2P) payment system. Secondly, this solution has been integrated with an autonomous (agent-based) grid management. The integrated solution of both marked platform as well as agent based control has been implemented and tested in a real microgrid with different distributed components such as PV System, CHP and different kinds of controllable loads. This microgrid is located in the distributed energy resources laboratory at the University of Reutlingen. Thirdly, the resulting solution is being implemented as an easy to customize market solution by AC2SG Software Oy, a Finland based software company, developing solutions for the Indian market. In a next phase, the solution is going to be tested in real environment in off-grids systems in India.
\end{abstract}

\section{Key words}

Distributed markets, distributed ledger technologies, blockchain, distributed energy resources, off-grid systems.

\section{Introduction}

In the last years the use of distributed ledger technologies (DLT) has been a matter of discussion in the energy sector, a good example of that is the DENA ${ }^{1}$ Study in 2014 [1]. It discussed the applicability and interest of this technology in the energy sector by means of surveys and interviews with key actors of the energy economy in Germany. Recently, the BDEW ${ }^{2}$ presented also an analysis of case

\footnotetext{
${ }^{1}$ DENA (Deutsche Energie Agentur) is the German

Energy Agency

${ }^{2}$ BDEW (Bundesverbands der Energie-

und Wasserwirtschaft) is the German Federal Association

of Energy and Water Management
}

studies and applicability of mainly Blockchain based DLTs in the energy sector [2]. This study provided also a mapping of current activities and application fields of Blockchain in Europe, US and the rest of the world. The perception of DLTs in the energy economy has evolved since 2014 to nowadays, from seeing Blockchain as a hype with no clear applicability to seeing Blockchain and in general DLTs as an "in some application cases" already mature technology, offering clear advantages in automation of processes.

\section{A. DLT in the energy economy}

Nowadays, the energy sector is clearly focusing on decentralization, decarbonisation and digitalisation. The decentralisation of the energy production has enabled a rising number of new actors such as the prosumers, meaning consumers who produce and consume energy at the same time, to enter the energy economy. The integration of these new actors in an energy economy marked by a tight straitjacket of processes and fixed relationships along a strong integrated value chain- is being very difficult. The digitalisation has also provided new solutions which are breaking down the traditional process boundaries and leveraging more dynamic relationships between the different players along the value chain. A clear example is found in enabling households and businesses to be increasingly active through small-scale interactions to participate in market processes. In this changing panorama, DLT (i.e. Blockchain) offers an interesting solution by making very small transactions economically viable, enabling direct trading between market players or even providing the means to "share investments", by for example supporting crowdfunding platforms.

Basically, Blockchain remove the necessity of an intermediary to validate a transaction. The technology behind is currently based on a proof-of-work (PoW) concept [3]. This requires a high amount of processing power, and therefore is not able to compute many Transactions per Second (TpS). Every computing data processor (miner) is competing with each other and 
trying to proof that its solution is the best. The PoW concept is the most decentralized and is sufficient for timeuncritical transactions. An up to 10 times faster alternative is the proof-of-stake (PoS) concept. In the PoS, the creator of the next block is chosen via various combinations of random selection and wealth or age (i.e. the stake). The last concept, proof-of-authority, can only be done centralized and in a private environment. A move from PoW to PoS is being observed as blockchain systems increase in complexity and speed needs, for example in the Ethereum Blockchain ${ }^{3}$, presented in Reference [4]. Every one of those concepts provide advantages and disadvantages depending on its application to the energy sector. Therefore, the choice of the Blockchain concept for the energy economy is depending on its application. Furthermore, there are obvious advantages in all those concepts compared to centralized systems including ITSecurity, transparency and cost-efficiency. An alternative to the use of blockchain based DLTs is the Directed Acyclic Graph (DAG) technology, such as Tangle. A comparison between both blockchain and blockchain-less technologies can be found on Reference [5]. The potential of Tangle is the lower use of system resources to find a consensus [6]. The Tangle ledger is claimed to be able to settle transactions with zero fees so devices can trade exact amounts of resources on-demand, as well as store data from sensors and dataloggers securely and verified on the ledger. Although there is very limited experience at the moment with Tangle in the energy economy.

Even while Blockchain is seen as an enabling technology for decentralisation and digitalisation, current blockchain based concepts are still far from significantly penetrate the market. Reference [2] claims that provided all the technical issues related for example to speed of the transactions or IT-security are resolved, the problem remains on profitability of the business case, on the acceptance of the population and on the regulatory aspects. In this last, the use of Blockchain is challenging the current general contract law, the energy law and the data privacy protection.

\section{B. Multi-Agent based control}

The majority of current hybrid systems (in microgrid configuration) have a central unit in charge of the management of the different components. This fact challenges the tolerance to faults as well as the scalability of the system. Adding a new component to the system implies an update to the management, which can be difficult when large microgrids with different players and different business interests are involved. Therefore, this paper will take another approach and decentralise the management in a new multi-agent based distributed system architecture. The use of multi-agent control has been a matter of discursion in the energy sector during the last decade, for example Reference [7] discussed agent-based computing and its relevance for control of power systems or Reference [8] proposes a multi-agent based distributed control architecture for energy management based on noncooperative game theory.

\footnotetext{
${ }^{3}$ More information about Ethereum blockchain under https://www.ethereum.org/
}

\section{Transactional control}

The transactional or transactive control is a new term coined to represent the coupling of accounting technologies with control strategies in power grids. As elaborated in Reference [9]: smart in-home devices, if integrated with the capability to transact energy with one another and with the electric grid, can offer a disruptive solution to managing energy supply and demand. Such transactive energy networks could turn homes from passive energy consumers into intelligent, active energy storage and service providers for the future grid. The transactive grid word was linked to the blockchain world in 2016, when the first P2P transaction occurred among neighbours in a microgrid who didn't have solar systems and those who produced excess solar electricity. This was the first application of blockchain for management and control purposes of a microgrid and it was successfully tested in Brooklyn, see the details on Reference [10].

\section{The concept}

The concept ${ }^{4}$ behind this work aims at developing a market solution that enables end customers of the energy system to participate in new local micro-energy-markets by providing them with a distributed, decentralized, transparent and secure Peer to Peer (P2P) payment system. This solution will be integrated with an autonomous microgrid management.

The concept and its application area is provided on figure 1.

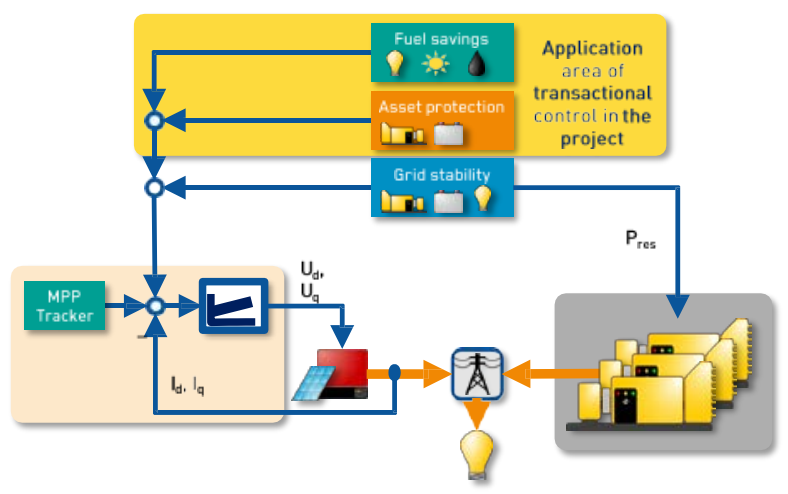

Fig. 1. Concept of this work and application area

The main achievements of the application of this concept are:

- $\quad$ Providing means to perform micro-transactions in a P2P fashion between end consumers and prosumers in local communities at low cost in a transparent and secure manner;

- Developing a standard platform with pre-defined smart contracts able to be tailored to different end customers'needs in an easy way;

${ }^{4}$ This concept was developed in a close cooperation between the finish small and Medium Enterprise/Small and Mid-sized Enterprise (SME) AC2SG Software Oy and the German Reutlingen University. 
- Developing the multi-agent based control for a microgrid (generation and loads) and;

- Integrating both the market platform as well as the local control of generation and loads.

The concept has been developed and tested in the microgrid laboratory at the Reutlingen University. The resulting development will be implemented in a market product and commercialized by the company AC2SG Software Oy, as a new product line which can be used for the control, market and accounting of small hybrid systems, mainly focussing the microgrids Indian market.

Furthermore, the idea is to extend the solution to accommodate a grid connected tenant application, allowing AC2SG to develop a product line for use cases in the European market.

\section{The test microgrid in the Reutlingen University}

The test microgrid is composed of different distributed generation resources and a group of loads, every one of them dotted with a distributed agent. Those agents are integrated in the DLT market platform, so that the control decisions are actually subordinated to the contractual decision algorithms implemented in the smart contracts. Furthermore, all the transactions will be recorded and be of public access for all the involved actors in order to increase the transparency of the management. The logic of the systems goes from decision making based on market signals every $15 \mathrm{~min}$ to implementing these decisions in real time thought the multi-agent based control. Bundling both of this layers and testing them in a real microgrid environment is the main contribution of this paper.

The virtual power plant Neckar-Alb laboratory ${ }^{5}$ together with other started projects such as the current prototype of the battery inverter REFUHybrid 100 has provided the necessary infrastructure to test the different strategies. Furthermore, this laboratory is connected to the Herman Hollerith center infrastructure to develop smart contract applications on the Ethereum Blockchain. This work has make used of both resources the microgrid and the blockchain infrastructure.

\section{A. Current test infrastructure}

The laboratory contains three different microgrids connected to the main grid and able to be configured with different components, such as CHP units, PV plant, batteries, charging stations for electromobility, district heating, thermal storage, etc.

\footnotetext{
${ }^{5}$ It was developed within the public funded project Virtual Power Plant Neckar-Alb (http://www.virtuelles-kraftwerkneckar-alb.de/ )
}

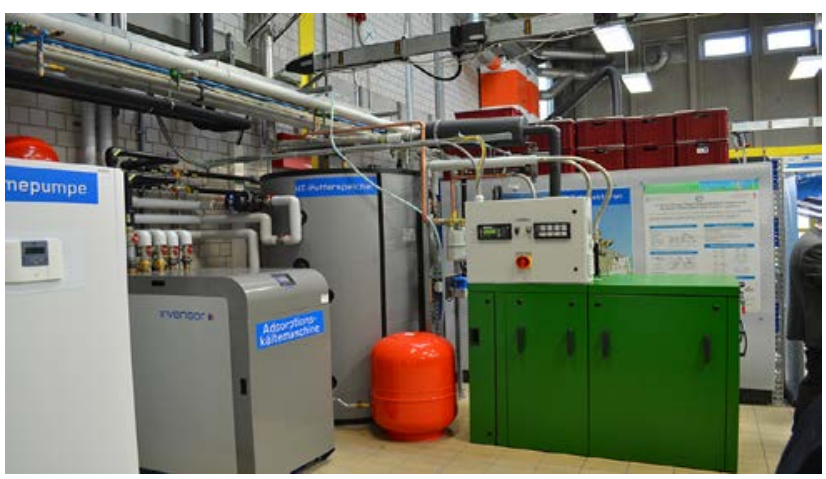

Fig. 2. microgrid components

The available power of the main components is about 12 $\mathrm{kW}_{\text {peak }}$ of Photovoltaic generation, $12 \mathrm{kWh}$ of the battery, 3 CHP Units with a $20 \mathrm{kWh}_{\mathrm{el}}$ installed power and different configurable loads. The microgrid components being used in this test are presented on figure 2 .

All the components were originally communicating with an EMS, able to perform optimisation process and schedule the microgrid based on 24 hours load schedule and weather forecast and different energy prices, see on figure 3 .

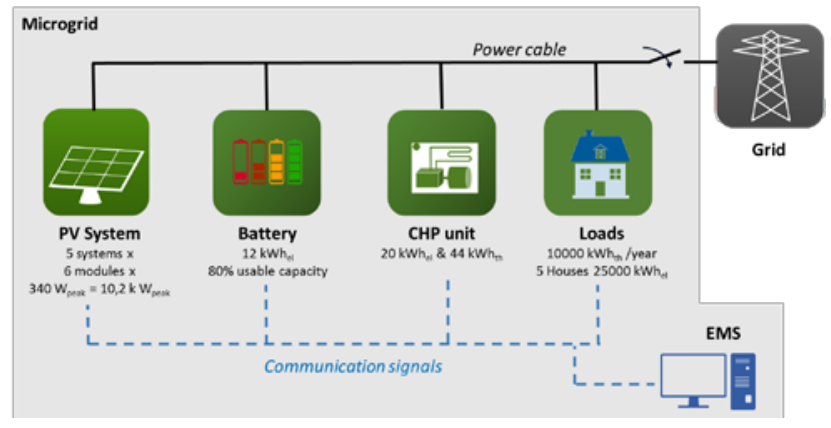

Fig. 3. Original microgrid configuration with central management

Currently, the various facilities are being equipped with Raspberry Pis for the programming of smart contracts. The IT infrastructure has been expanded and connected to the Herman Hollerith Blockchain. In order to ensure the economical and resource-saving operation of the laboratory, large loads will only be simulated by using one or more regenerative load simulators, where the consumed energy is not to be converted into heat, but to be fed back into the grid.

\section{DLT based solution for the local market platform}

The DLT used in the implementation is the Ethereum Blockchain and the logic of the market has been designed in the smart contracts. The market rules have been programed using Solidity, a DLT language based on java Script, see main components on figure 4. 


\section{A. The Market Design}

Two strategies have been implemented for the market design:

- $\quad$ P2P transactions between the network participants using a single smart contract. It basically works like a market place.

- A single smart contracts for each network participant. In this concept several smart contracts interact with each other.

The private Blockchain at the Reutlingen University (Herman Hollerith center) has been used to test both solutions. The two solutions were proposed and developed in a Hackathon in the Reutlingen University. The results of this Hackathon are available online ${ }^{6}$.

Within the first strategy, the Smart Contract is deployed via Mist browser to the private Ethereum blockchain. It represents a trading platform, which can be used by the different actors. They can perform the following activities: Charge their prepaid account with ether; Offer energy for a specified ether price or; Buy a specific amount of energy for the offered ether price. Each of these activities are realized by a function of the Smart Contract. To be able to run these functions, the Smart Contracts ether wallet is loaded with the total prepaid balance of all actors. Different account variables (representing the different stored prepaid balance of each market participant) are defined and contain the offers of each involved actor. The logic for the separation is implemented in the charging and buying function. The logic of the smart contract is presented in figure 5 .

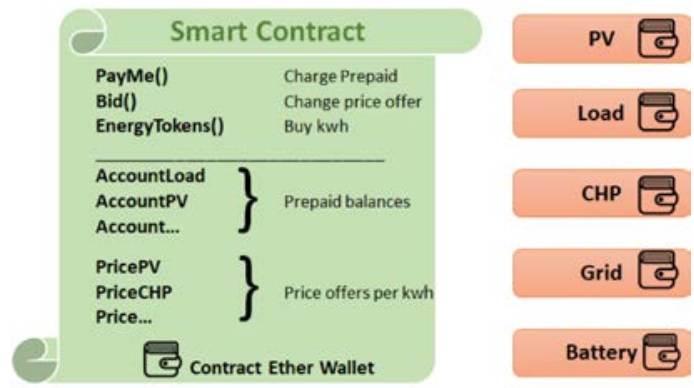

Fig. 5. Logic of the Smart Contract

Within the second strategy, the stakeholder Load owner transfers ethereum to the smart contract and the other nodes send energy in form of energy tokens as exchange to the load. The smart contract acts like an escrow and holds the transferred amount of ethereum until the Load owner confirms the arrival of the item. After the confirmation the transferred amount of ether will be released to the other stakeholders.

\section{Autonomous (multi-agent-based) grid management}

The multi-agent-based control has been implemented in the different components of the microgrid and linked to the DLT nodes. The control's objective is to regulate the power flows to and from the main grid. This is done by collecting all actors' power production and consumption in the state variables of a smart contract. The control algorithm calculates the maximum possible injection/consumption, which is then read by the generators and loads.

This approach can also be combined to register energy flows from the different components and allow for immediate, seamless payment of the energy. If variable pricing of sources is implemented (future), the Blockchain would allow for a transparent accounting on the time, amount and price at which each transaction took place.

\section{Tested Use Case}

The use case being tested in the microgrid at the Reutlingen university is presented on figure 6. The components building up the microgrid in this case are a PV System, a battery, a CHP unit and loads.

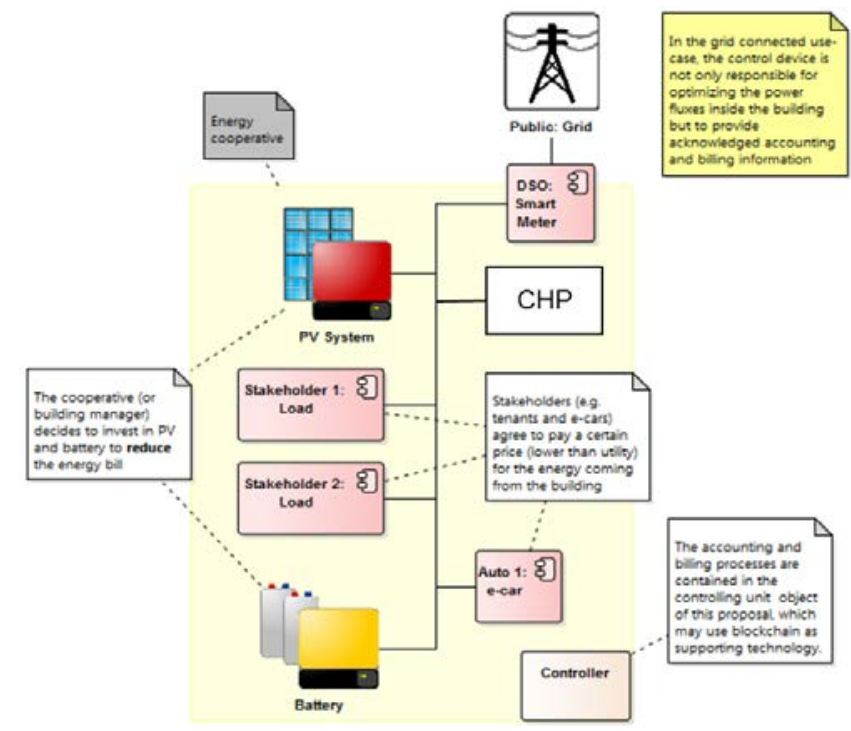

Fig. 6. Tested use case

The current results of the test are preliminary.

The transaction agreed in the smart contract, determines the kWh every $15 \mathrm{~min}$ produced and consumed by every component. This information is sent to the control agents. At this moment, the control strategy is based on maximizing the production with $\mathrm{PV}$ and adapting the production of the CHP unit to the heat requirements. The initial bid of the components is for PV Energy 20 $\mathrm{Ct} / \mathrm{kWh}$, for the CHP produced electrical energy 25 $\mathrm{Ct} / \mathrm{kWh}$ and the price of the energy bought from the grid $30 \mathrm{Ct} / \mathrm{kWh}$. The results of the market clearance developed in the smart contracts is provided on figure 7 . 


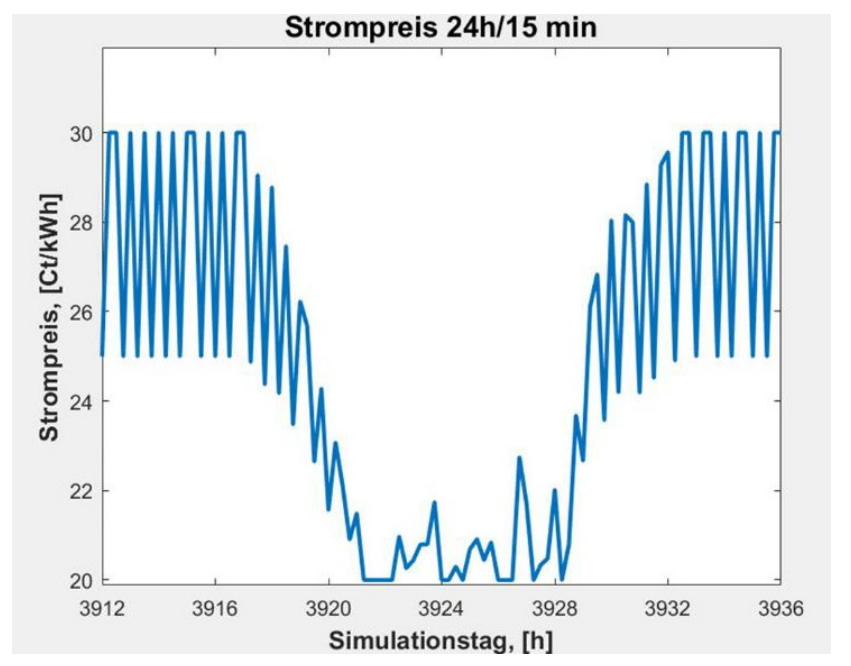

Fig. 7. Market clearing price calculated with the smart contracts

The market strategy has been tested in the Ethereum test net Rinkeby and takes an average time to create a block and validate a transaction of $14 \mathrm{~s}$. By using a test blockchain, the number of transactions are isolated from the price of the gas. Therefore, the price of actually performing the transaction has not been taken into consideration.

The agents have been tested with matlab models of the different components in a hardware in the loop configuration, the agents as well as the nodes of the blockchain are integrated in raspberry pis and connected to the test blockchain. The next step is to test it with the real components and connected to the private blockchain available at the Herman Hollerith center,

\section{Conclusion}

This paper has presented a solution to integrate DLT in the multi-agent control of a microgrid. The development of the market strategies has been programmed in the smart contracts and deployed in an Ethereum test Blockchain. This had the advantage of isolating the transactions fees from the real price of the gas. The market allows transactions every $15 \mathrm{~min}$ and the agents maintain the stability of the system within those periods.

At the moment, the results of the transactions have been tested with the matlab models of the different components of the microgrid.

\section{Acknowledgement}

The authors wish to acknowledge the Virtual Power Plant Neckar-Alb as well as the Herman Hollerith center to allow the use of their installations.

\section{References}

[1] Burger, A. Kuhlmann, P. Richard, J. Weinmann, "Blockchain in the energy transition. A survey among decisionmakers in the German energy industry”, Deutsche Energie-Agentur GmbH (dena) - German Energy Agency Energy Systems and Energy Services, Page 12, Nov. 2016

[2] BDEW Bundesverband der Energie- und Wasserwirtschaft e.V., „Blockchain in der Energiewirtschaft - Blockchain in the energy sector“, K08, Page 63, Oct. 2017

[3] Nakamoto, S. Whitepaper „Bitcoin: A Peer-to-Peer Electronic Cash System”, 2009.

Downloadable from: https://bitcoin.org/bitcoin.pdf

[4] V. Buterin, "Ethereum: A Next-Generation Cryptocurrency and Decentralized Application Platform" Bitcoin Magazine. 23. Januar 2014.

[5] K. Yeow, A. Gani, R. W. Ahmad, J. J. P. C. Rodrigues and K. KO, "Decentralized Consensus for Edge-Centric Internet of Things: A Review, Taxonomy, and Research Issues," in IEEE Access, vol. PP, no. 99, pp. 1-1. doi: 10.1109/ACCESS.2017.2779263

[6] Serguei Popov, “IOTA Whitepaper: The Tangle” 2017. Online available: https://iota.org/IOTA_Whitepaper.pdf Last Visited on 29.01.2018

[7] N. R. Jennings and S. Bussmann, "Agent-based control systems: Why are they suited to engineering complex systems?," in IEEE Control Systems Magazine, vol. 23, no. 3, pp. 61-73, June 2003.

[8] M. Reyasudin Basir Khan, R. Jidin, J. Pasupuleti, "Multiagent based distributed control architecture for microgrid energy management and optimization”, Energy Conversion and Management, Volume 112, 2016, Pages 288-307, ISSN 0196-8904.

[9] N. Wang, "Transactive control for connected homes and neighbourhoods". Nature, Energy volume 3, pages 907909 (2018)

[10] H. Breuer, “A Microgrid Grows in Brooklyn”, Siemens newsletter, Jan. 2017. Downloadable from https://www.siemens.com/innovation/en/home/pictures-ofthe-future/energy-and-efficiency/smart-grids-and-energystorage-microgrid-in-brooklyn.html 